\title{
Structure formation in a binary monolayer of dipolar particles
}

\author{
I. Varga, ${ }^{1}$ H. Yamada, ${ }^{2}$ F. Kun, ${ }^{1, *}$ H.-G. Matuttis, ${ }^{2}$ and N. Ito ${ }^{3}$ \\ ${ }^{1}$ Department of Theoretical Physics, University of Debrecen, P.O. Box 5, H-4010 Debrecen, Hungary \\ ${ }^{2}$ The University of Electrocommunications, Department of Mechanical Engineering and Intelligent Systems, Chofu Chofugaoka 1-5-1, \\ Tokyo 182-8585, Japan \\ ${ }^{3}$ Department of Applied Physics, School of Engineering, The University of Tokyo, Bunkyo-ku, Tokyo 113-8656, Japan
}

(Received 5 January 2005; published 31 May 2005)

\begin{abstract}
We propose an experimental technique for an easy to control realization of a binary dipolar monolayer where the two components have oppositely oriented dipole moments constrained perpendicular to the plane of motion without the application of an external field. The experimental setup ensures that hydrodynamic effects do not play a crucial role in the structure formation, the particles move deterministically due to the dipole-dipole interaction. At low concentrations, cluster-cluster aggregation occurs with chainlike morphologies, while at high concentration the particles self-assemble into various types of binary crystal lattices, in good agreement with the theoretical predictions. The structures formed by the particles are found to be sensitive to external perturbations due to the central interparticle forces, however, static friction arising at the contact surface of particles can increase the stability compared to systems with only viscous friction.
\end{abstract}

DOI: 10.1103/PhysRevE.71.051405 PACS number(s): 82.70.Dd, 83.10.Pp, 41.20. - q, 61.46. + w

\section{INTRODUCTION}

In a monolayer of magnetic particles, the long range anisotropic interaction and the inherent frustration of the system give rise to very interesting ordering phenomena and pattern formation [1-24]. During the last decade this pattern formation has been investigated through various experimental techniques with particle sizes from the micrometer to the centimeter range. In the experiments, the particles are usually suspended in an electromagnetically passive viscous liquid or are confined to the liquid-air or liquid-liquid interface. Investigations have been performed with several different types of particles subjected to constant or time-dependent external fields. While in the absence of external fields, chains, rings and fractal clusters have been observed with a temperature-dependent fractal dimension [1-12]; in the presence of an external field crystal structures emerged [13-24]. It was demonstrated that by an appropriate adjustment of the external field all planar lattice structures can be produced [13-25], which is of high practical importance for the controlled fabrication of patterned surfaces; and the essential problems of statistical physics like the study of phase transitions between different morphologies and the phenomenon of melting in two dimensions [13-16] are also addressed.

A very interesting type of structure formation occurs in a dipolar monolayer when the dipole moment of particles is constrained to be perpendicular to the plane of motion. The two possible orientations of the dipole moments distinguish two components of the system that can also differ in other physical properties like the magnitude of the dipole moment, material type, size, mass, etc., so that the system is also termed a binary colloid. A possible physical realization of binary dipolar monolayers was recently presented in Ref. [26], where particles of two different material properties

\footnotetext{
*Electronic address: feri@dtp.atomki.hu
}

were sedimented in an electromagnetically passive liquid. The monolayer was subjected to an ac electric field perpendicular to the bottom plate of the container, so that the particles attain an induced dipole moment parallel to the field. Under the action of the long range dipolar interaction, the particles start to move and form various structures, depending on the parameters of the experimental setup. Novel behavior was obtained in the parameter range where particles of a different type have oppositely oriented dipole moments attracting each other. At low concentrations binary chains, rings, and polygons of alternating particles were observed, while at high concentrations the particles self-assemble into binary crystal lattices [26].

Recently, we have carried out a theoretical study of the self-assembly of particles in a binary colloidal monolayer [27]. Our model assumed that the dipole-dipole interaction dominates the behavior of the particle ensemble and thermal motion of particles does not occur due to the large particle mass. Furthermore, the carrier liquid overdamps the motion of particles, trapping the system in local energy minima. Molecular dynamics simulations and analytic calculations showed that the structure of the monolayer is determined by the total concentration of the particles, the relative concentration, and the relative dipole moment of the components. At low concentrations kinetic aggregation occurs, giving rise to chains, closed loops, and fractal structures of alternating particles. Increasing the concentration, a crossover takes place from chainlike to more compact branching morphologies characterized by a higher value of the fractal dimension. At high concentrations various types of crystal structures are obtained in parameter regions, which are in good agreement with the experimental results [26]. However, several predictions of our model had not been realized experimentally in Ref. [26]: the dynamics of the aggregation process, the fractal character of the growing aggregates, the formation of the binary honeycomb lattice where both types of particles have three neighbors of the other type, or the occurrence of quasicrystalline order with local fivefold symmetry were not ob- 


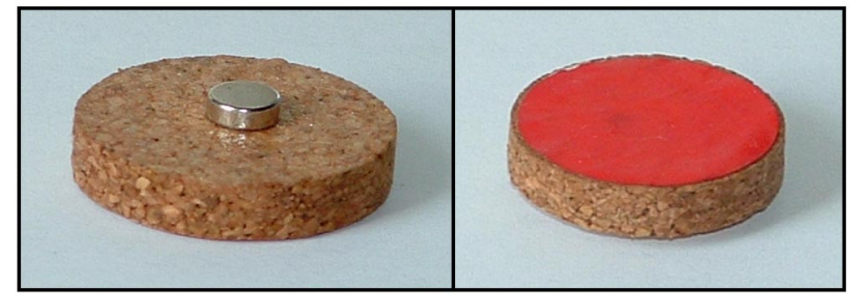

FIG. 1. (Color online) Magnetized particle attached to a cork disk of larger diameter (left). The composite particle can float upside down on the surface of water (right). The diameter $d$ and height $h$ values are $d=4 \mathrm{~mm}$ and $h=1.5 \mathrm{~mm}$, and $d=26 \mathrm{~mm}$ and $h$ $=5 \mathrm{~mm}$, for the magnetic particle and cork disk, respectively.

served. It was reported in the experiments that the driving field induced electrohydrodynamic (EHD) flow of the embedding liquid, which brings adjacent particles together. This EHD effect, on the one hand, facilitates the aggregation of particles; on the other hand, however, it can have a strong influence on the structures attained by the particle system. The question naturally arises if the discrepancy of the experimental results [26] and model calculations [27] is due to the EHD flow.

In this paper we present an experimental study of the structure formation in a binary monolayer of particles. We propose an experimental method that provides a straightforward, easy to control realization of binary monolayers without an external driving field. The two components of the system are millimeter-sized particles with permanent magnetic dipole moment whose orientation is fixed to be perpendicular to the plane of motion. To decrease the frictional barrier, the particles are confined to a liquid-air interface, i.e., the particles float to reduce the friction with the surroundings. The setup ensures that hydrodynamic effects cannot play a crucial role, the particles undergo deterministic motion due to the dipole-dipole interaction, furthermore, the liquid makes the system overdamped so that the experimental results can be better compared to the theoretical ones. Excellent agreement is obtained between the experimental results and the theoretical predictions of Ref. [27].

\section{EXPERIMENTAL SETUP}

Our experimental setup provides a simple way to study the structure formation in a binary dipolar monolayer. In the experiments, macroscopic metal particles of cylinder shape are magnetized along their axis. In order to facilitate the particle motion, the magnetic particles are attached to cork disks of regular shape so that the composite particle can float on the surface of a liquid. Figure 1 illustrates that the two particles are adjusted on the top of each other to have a common axis, which ensures stability when floating. In all experiments, water was used as a carrier liquid to which a surfactant was added to reduce the surface tension. The particles are placed onto the water surface with the heavier part pointing downward, i.e., the magnets are always under the water; see Fig. 1. In the experiments, magnetic particles of three different strengths were used with slightly different geometrical extensions attached always to cork disks of the

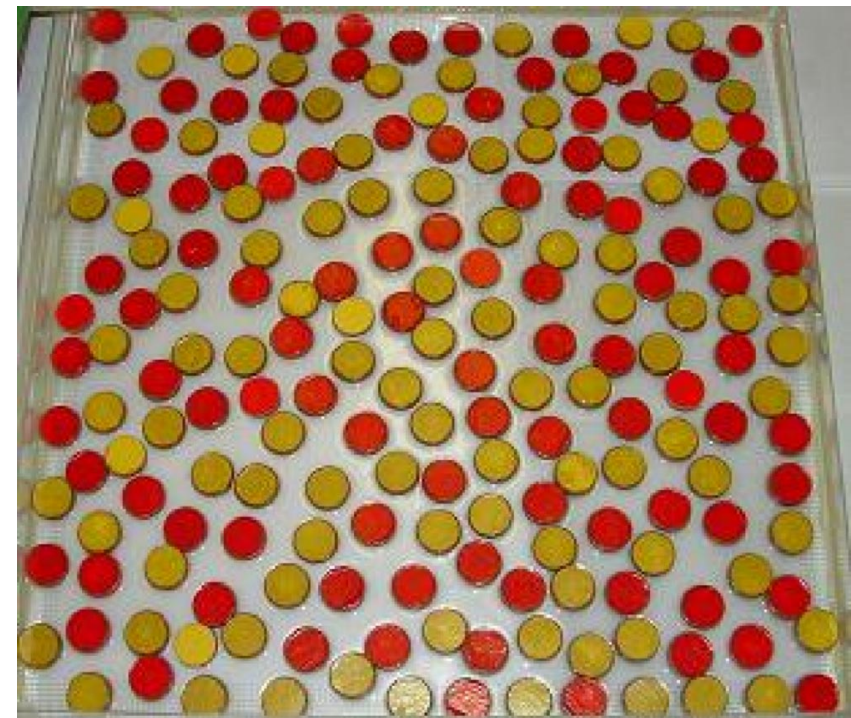

FIG. 2. (Color online) Initial configuration of 176 particles in a square box of side length $L=500 \mathrm{~mm}$, resulting in a concentration $\phi \approx 0.35$. The same amount of particles of opposite magnetic moment/different colors is used, so that $\phi_{r}=1$. The water level is low enough to prevent floating of the particles.

same size. The cork disks have diameter $d=26 \mathrm{~mm}$ and height $h=5 \mathrm{~mm}$, which proved to be sufficient to prevent the flipping of magnets of identical orientation in the vicinity of each other, even for the largest magnetization used in the present study.

The two components of the system are realized by the two opposite orientations of the dipole moments of the particles perpendicular to the water surface. The initial state is prepared such that the particles are placed into a square-shaped container of side length $L=500 \mathrm{~mm}$ when the container contained no or only a thin layer of water, not enough to float the particles. The initial particle positions had been generated beforehand by a computer program and the particles were positioned by hand accordingly, making use of a centimeter grid under the transparent container (see Fig. 2). The free side of the cork disks is colored according to the orientation of the dipole moments of the magnetic particles, i.e., the red color (dark particle) in Fig. 2 indicates dipole moment pointing upward perpendicular to the water surface, while yellow (light particle) stands for downward dipoles. Starting from the random initial configuration, the water level is raised very slowly in order to ensure that all the particles start floating approximately at the same time. Then the floating particles move under the action of their mutual dipole-dipole interaction and form various structures.

It is interesting to note that the ensemble of millimeter- or centimeter-sized particles having a permanent magnetic moment can be considered as a cohesive granular material with a long range anisotropic cohesion force. Recent observations revealed that vertically vibrated magnetic spheres can selforganize into clusters, varying the vibration intensity with respect to the strength of the dipolar interaction [28]. Magnetic granular materials of two components were found to show interesting segregation phenomena just due to differences of the strength and the shape of the dipole field of the 
components $[29,30]$. It has been shown experimentally and theoretically that the anisotropic cohesive force also changes the distribution of avalanche sizes when particles are piled [25]. Similar to the experiments on magnetic granular materials $[12,28-30]$, a hard vibrating table could also be used to excite the particles for binary monolayers to overcome the frictional barrier with the bottom plate. However, this technique would also give rise to fluctuations of the direction of dipoles, which could have a strong effect on the final result, especially when crystalline order is searched for. Twodimensional crystal structures of millimeter-sized particles have been recently obtained on a vibrating table by charging spherical plastic particles using triboelectricity methods [15], however, this study was restricted to the simplest case of the Wigner crystal.

The total concentration $\phi$ of the particles in our experimental box is defined as the coverage,

$$
\phi=\frac{N R^{2} \pi}{L^{2}}
$$

where $N$ and $R$ denote the number and radius of cork disks carrying the magnetic particles, respectively. The partial concentrations of the two components $\phi_{1}$ and $\phi_{2}$ can be analogously defined and their relative concentration $\phi_{r}$ follows as

$$
\phi_{r}=\frac{\phi_{1}}{\phi_{2}} .
$$

Experiments were performed by varying the concentration $\phi$ and the relative concentration $\phi_{r}$ in a broad range by controlling the number of particles $N$ while the container size $L$ is fixed. In contrast to previous experiments [26], no external field is necessary in our setup, and the magnitude of the dipole moment is controlled by the placement of particles with the corresponding magnetization.

\section{MOTION OF PARTICLES}

The experimental setup ensures that the motion of particles is deterministic since the thermal motion is suppressed by the relatively large particle mass. Assuming that the magnetic properties of the particles can be represented by assigning pointlike dipole moments to their center, the force between two particles $i$ and $j$ floating on the water surface at relative position $\vec{r}_{i j}$ with parallel dipole moments of magnitudes $\mu_{1}, \mu_{2}$ can be cast in the form

$$
\vec{F}_{i j}^{d d}=3 \frac{\vec{\mu}_{1} \vec{\mu}_{2}}{r_{i j}^{4}} \frac{\vec{r}_{i j}}{r_{i j}} .
$$

The force in Eq. (3) is central, it falls always in the line connecting the two particles, and it is attractive (repulsive) for particles with opposite (parallel) dipole moments. The effect of the fluid on the particles is characterized by the Stokes drag, i.e., the force exerted by the fluid $\vec{F}_{i}^{s}$ on particle $i$ is assumed to have the form

$$
\vec{F}_{i}^{s}=-\alpha \vec{v}_{i},
$$

where $\vec{v}_{i}$ denotes the velocity of the particle relative to the fluid and $\alpha$ is the drag coefficient.
As a first step of the experiments, the motion of a particle in the dipole field of another particle of the same shape but opposite dipole orientation has been measured and compared to simple analytic calculations based on the interactions defined above. In these tests one particle was fixed while another particle with opposite dipole orientation was released from a distance $x_{0}$ with zero initial speed $v_{0}=0$. Both particles were initially placed far from the container walls and surfactants had been added to the water to minimize the effect of surface tension. The equation of motion of the released particle is obtained as

$$
m \frac{d^{2} x}{d t^{2}}=F_{i j}^{d d}-\alpha \frac{d x}{d t},
$$

where $m$ denotes the entire mass of the moving particle. Assuming that the system is fully dissipative and noninertial, Eq. (5) can be reduced to a first-order differential equation for the particle position $x$, with the solution

$$
t=\frac{\alpha}{3 \mu_{1} \mu_{2}}\left(x_{0}^{5}-x^{5}\right) .
$$

The time needed for the moving particle to reach the stationary particle can be determined by setting $x=d$ in the above equation, where $d$ is the diameter of the cork disk. In the experiment, the particle motion was recorded by a digital camera with a distance scale in the foreground, from which kinematic quantities could easily be determined. The time $t$ as a function of distance $x$ was measured for magnetic particles of two different extensions $d=2 \mathrm{~mm}, h=6 \mathrm{~mm}$ (more barlike), and $d=4.0 \mathrm{~mm}, h=1.5 \mathrm{~mm}$ (more disklike), with the same arrangement of swimmers. For the two particle arrangements, two sets of measurements were performed: in the first experiment the particles were floating on the water surface, while in the second one, the particles were placed inside a closed container and the external pressure was adjusted to keep the moving particle floating under the liquid surface. In order to compare the experimental results to the theoretical prediction of Eq. (6), the measured data were fitted to the curve,

$$
t=-a\left(x^{-b}+c\right),
$$

where $a, b$, and $c$ are fitting parameters. The experimental results together with the best fits obtained are presented in Figs. 3 and 4. Error bars are not shown in the figures since they are of the same size as the symbols.

It can be observed in the figures that although the motion of particles on the surface and inside the liquid set in approximately at the same initial distance $x_{0}$, the time values $t$ obtained are significantly higher for surface motion. This effect is caused, for instance, by the surface waves generated by the moving particle which results in an additional energy loss and slower motion. However, for both types of motion the experimental results can be well fitted by the functional form of Eq. (7). The best fits were obtained with the parameter values $b=5.05$ and $b=4.96$ in Fig. 3, and $b=5.18$ and $b=5.14$ in Fig. 4 for surface and bulk motion, respectively. Comparing the two figures, it can be noted that the fit has a somewhat better quality for particles with a more elongated 


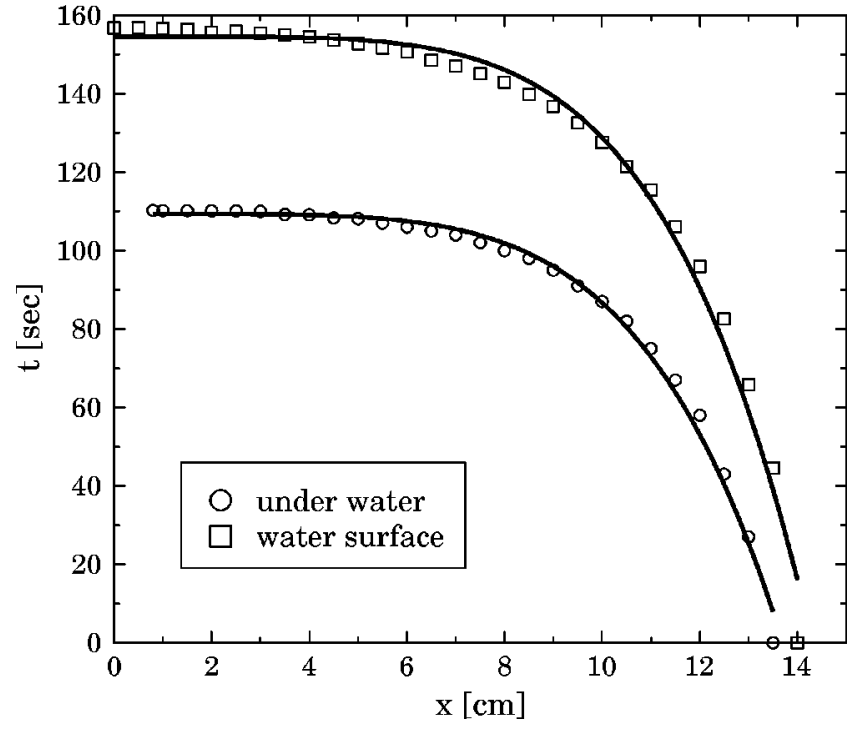

FIG. 3. The time $t$ as a function of distance $x$ measured during the motion of a prolate magnetic particle with extensions $d$ $=2 \mathrm{~mm}$ and $h=6 \mathrm{~mm}$. The solid lines show the best fits obtained with Eq. (7) for both moving on and under the water surface.

(bar) shape than for the one with disk-shaped dipoles, since in this case the pointlike dipole approximation provides a more accurate description of the magnetic field. The satisfying values of $b$ obtained by the fitting near the predicted value of 5 imply that the point-dipole approximation and the Stokes drag provide a reasonable description of the dynamics of the system for both particle shapes. Induced mass effects for the acceleration, as well as corrections to the dipole potentials, could be neglected.

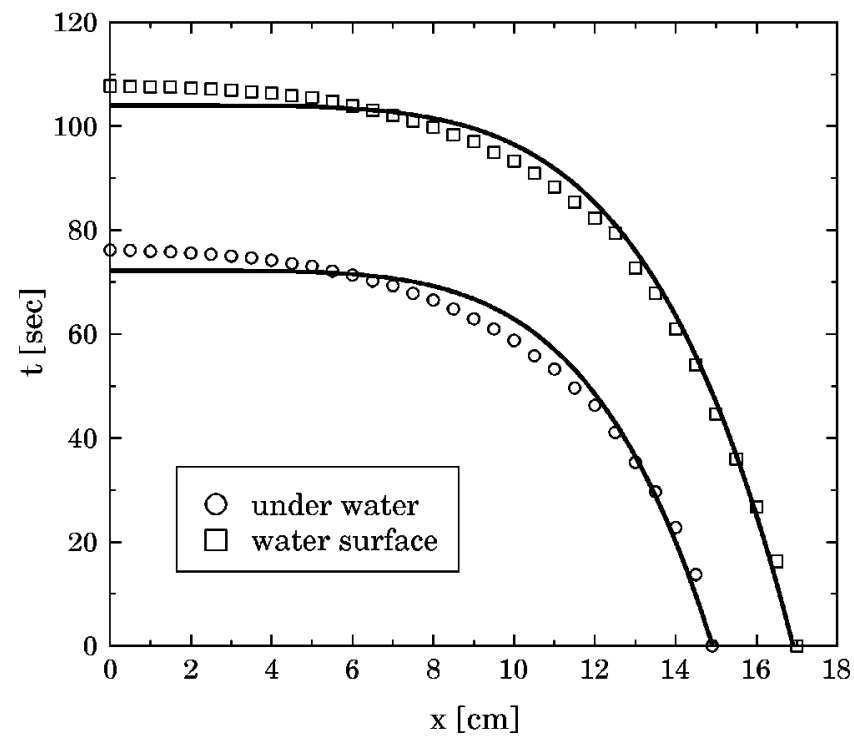

FIG. 4. Time $t$ as a function of distance $x$ measured during the motion of an oblate magnetic particle of diameter $d=4.0 \mathrm{~mm}$ and height $h=1.5 \mathrm{~mm}$ attached to a cork disk. The solid lines indicate best fits obtained with the formula, Eq. (7).

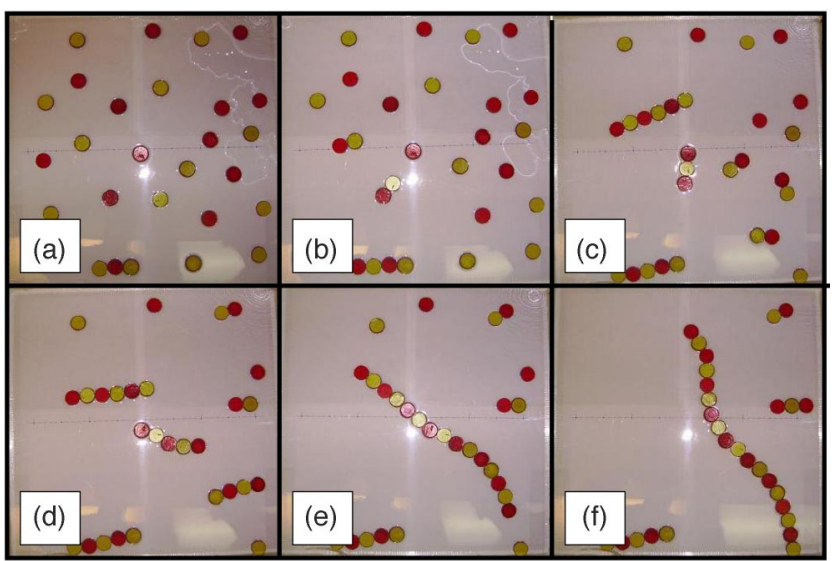

FIG. 5. (Color online) Cluster-cluster aggregation at low concentration $\phi \approx 0.05, \phi_{r}=1$, and $\mu_{r}=1$. Magnified clipping of a part of the experimental box is shown. The first single particles form short chains (a), (b), (c), which then merge into longer chains (d), (e), (f). Snapshots taken at times $t=1 \mathrm{~s} \mathrm{(a),} 10 \mathrm{~s}$ (b), $30 \mathrm{~s}$ (c), $50 \mathrm{~s}$ (d), $100 \mathrm{~s}(\mathrm{e}), 200 \mathrm{~s}$ (f).

\section{STRUCTURE FORMATION}

We carried out a large amount of experiments, varying the total concentration $\phi$ of the particles, the relative concentration $\phi_{r}$, and relative dipole moment $\mu_{r}$ of the components. In all these experiments, the disk-shaped magnetic particles were used, and the relative dipole moment $\mu_{r}$ of the components was controlled by using magnetic particles with the appropriate value of magnetization. In order to keep the particle number large, even at low concentrations, a container of side length $L=1000 \mathrm{~mm}$ was also constructed, in which, for instance, the concentration $\phi=0.05$ is realized by 102 particles.

At low concentrations we observed that due to the long range dipolar interaction, the particle system undergoes a cluster-cluster aggregation process, i.e., first single particles of opposite dipole orientation join and form small clusters which then further aggregate step by step until the entire system is covered by a network of particles. The time evolution of the cluster-cluster aggregation process is illustrated in Fig. 5, where part of the experimental box was cut out and magnified. The binary clusters of particles with oppositely oriented dipole moments can easily be identified in the figure. The cluster morphology is always chainlike, i.e., short chains merge at their ending points forming longer chains. This aggregation mechanism was found experimentally to work until the concentration is lower than $\phi^{*} \approx 0.1-0.15$. Above $\phi^{*}$, due to the relatively small distance of clusters, aggregation occurs not only at chain ends but also at internal sites, making the clusters more compact with branching morphology. The dynamics of the aggregation process and the structure of growing aggregates are in good qualitative agreement with the results of computer simulations of binary monolayers [27]. A quantitative characterization of the kinetic aggregation process of the binary system can be obtained by analyzing the average cluster size and the number of clusters as a function of time, and furthermore, the scaling structure of the cluster size distribution functions $[1,2,31]$. 


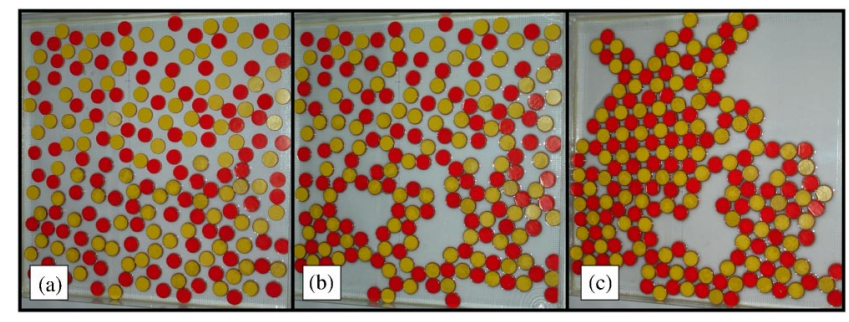

FIG. 6. (Color online) Time evolution of the pattern formation starting from an initially random configuration of 200 particles (a). When the water level was slowly increased, the particles start to move (b) and quickly self-assemble locally into a square lattice structure (c). The duration of the entire aggregation process was about $60 \mathrm{~s}$.

The results will be presented in a future publication along with large-scale computer simulations [32].

In the high concentration phase $\phi>\phi^{*}$, experiments were performed by varying the relative concentration $\phi_{r}$ and relative dipole moment $\mu_{r}$ of the two components. The total number of particles was fixed to 200 , which provides concentration $\phi \approx 0.4$ in the box of side length $L=500 \mathrm{~mm}$. Larger concentrations were achieved by mounting internal walls inside the container. To explore the parameter regimes of the occurrence of crystal structures, the experiments were repeated 5 times under identical conditions. A representative example of the pattern formation can be seen in Fig. 6 for a system of total concentration $\phi=0.4$, relative concentration $\phi_{r}=1$, and relative dipole moment $\mu_{r}=1$. When the water level is high enough, the particles get mobilized [Fig. 6(b)] and rapidly aggregate into compact structures [Fig. 6(c)]. We observed that the structure of clusters is not frozen, i.e., due to the isotropic interparticle forces, the cluster structure is quite flexible, in contrast to particle monolayers with inplane dipoles, where the anisotropic dipolar interaction makes the clusters more rigid [11]. In our case the flexibility has the consequence that when two clusters approach each other, they deform, facilitating the aggregation. Furthermore, the subsequent aggregation steps can result in rearrangements of particles reaching deeper energy minima. Typically, the perturbation of an aggregate by a particle, joining the cluster with a non-zero velocity, may give rise to an avalanche of restructuring events. The square lattice presented in Fig. 6(c) was obtained as a result of several restructurings. Similar rearrangements of particles inside clusters have also been observed in simulations of binary monolayers [27].

Figure 7 summarizes the crystal structures obtained experimentally in the binary monolayer, furthermore, Table I provides an overview of the corresponding parameter regimes. When the two components of the system have a dipole moment of the same magnitude, the binary square lattice provides the lowest energy configuration [27]. Experiments showed that under the condition $\phi_{r}=1$ and $\mu_{r}$ $=1$ the square lattice is always obtained for high enough concentrations $\phi \geqslant 0.4$ [see Fig. 7(a)], in good agreement with the analytic calculations and computer simulations of Ref. [27]. For concentrations in the interval $\phi \approx 0.2-0.4$, the particles form short chains that then join into a network structure with local crystalline order. It is important to em-

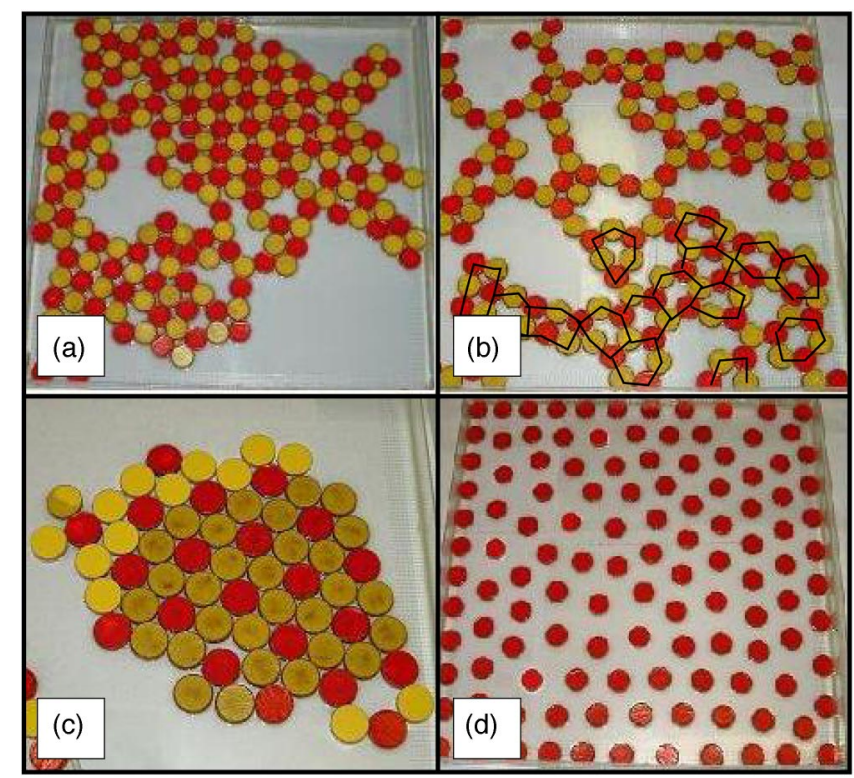

FIG. 7. (Color online) Overview of crystal structures obtained in the experiments. (a) Square lattice occurring at $\phi_{r}=1, \mu_{r}=1$, and $\phi \approx 0.4$. (b) Honeycomb lattice obtained at $\phi_{r}=1, \mu_{r}=1$, and $\phi$ $\approx 0.35$. The honeycomb structure is highlighted by black lines. (c) HCP structure at $\mu_{r} \approx 2.5$, and $\phi_{r}=2$ obtained in a region of the container when the local concentration was about $\phi=0.7$. (d) Triangular lattice occurring when the system consists exclusively of one type of particles.

phasize that in this concentration regime, locally binary honeycomb lattice structures were obtained where particles of both types have three neighbors of opposite dipole orientation [see Fig. 7(b)]. Our experiments showed that the binary honeycomb lattice can occur for concentrations $\phi \approx 0.3$ -0.4 with $\phi_{r}=1$ and $\mu_{r}=1$. However, in computer simulations an asymmetry, i.e., $\mu_{r} \approx 2.5$ of the two components, was required to make the honeycomb lattice energetically more favorable than the square lattice structure. In computer simulations, a relatively small irregularity of the honeycomb structure at $\mu_{r}=1$, as for the one presented in Fig. 7, would initiate a collapse into a square lattice, achieving a deeper energy. This discrepancy between the experimental and theoretical results can be attributed to the static friction in the experiment at the contact surface of the particles, which stabilizes the structures like in the packing of granular materials. Static friction was also found to have an important sta-

TABLE I. The parameter regimes determining the final structure of the system. For an explanation, see the text. (In the first row missing values of $\phi_{r}$ and $\mu_{r}$ indicate that the system consists exclusively of one component.)

\begin{tabular}{cccc}
\hline \hline Structure & $\phi$ & $\phi_{r}$ & $\mu_{r}$ \\
\hline Triangular latt. & $0-0.9$ & - & - \\
Chains & $0-0.15$ & 1 & 1 \\
Square lattice & $\geqslant 0.40$ & 1 & 1 \\
Honeycomb & $0.30-0.40$ & 1 & 1 \\
Binary HCP & $\geqslant 0.7$ & 2 & $2.5-$ \\
\hline \hline
\end{tabular}


bilizing effect in experiments on magnetic monolayers with in-plane dipole moments [11]. In the computer simulations, no static friction was implemented [27], so the central interparticle forces allowed a rearrangement of the whole system. In the experiments of Ref. [26], the honeycomb lattice was not observed. However, it was argued that the electrohydrodynamic flow of the embedding liquid plays an important role in the structure formation, namely, it brings the particles in the vicinity of each other. Our experiments showed that such a flow can also generate strong enough perturbation to destroy the loose structure of the honeycomb lattice.

If one of the components has a much larger magnitude of dipole moments than the other one, binary hexagonal closed packed (HCP) structures can be obtained in the limiting case of high concentrations $\phi \geqslant 0.7$. Such high concentrations were achieved in our experimental setup by reducing the container area by mounting internal walls of the container. Experiments showed that very high concentrations, close to the highest coverage, are unfavorable for structure formation because they freeze the particles in the initial state. The binary HCP lattice presented in Fig. 7(c) was obtained at $\mu_{r}$ $\approx 2.5, \phi=0.7$. Compared to the simulation results, the concentration $\phi$ had to be lowered since at higher concentrations the static friction of the particles stabilized the system almost in the initial configuration. In the HCP lattice, the particles of the larger dipole moment are encircled by 6 particles of the other type, however, only 3 particles with large dipole moment surround the smaller one, so that the relative concentration was chosen to be $\phi_{r}=2$.

If the system consists exclusively of particles of the same orientation, due to the repulsive interaction of parallel dipoles, the particles order in a triangular lattice, as illustrated in Fig. 7(d). The particles line up along the rigid walls of the container which produces a strong distortion of the symmetry of the system so that regular triangular structures are mainly observed in the middle of the container; see Fig. 7(d).

\section{DISCUSSION}

We presented an experimental study of the self-assembly of particles in binary dipolar monolayers. We constructed an experimental technique which provides a straightforward and controllable realization of binary monolayers with particles of oppositely oriented dipole moments constrained to be perpendicular to the plane of motion. In the experimental setup, macroscopic particles are constructed by attaching particles of a permanent magnetic moment to swimmers, which ensure the confinement of the composite particles to the airwater interface (floating), and prevent flipping, constraining the dipole moment of the particles perpendicular to the water surface. One of the main advantages of the experimental setup is that it does not require external fields to generate particles of different dipole moments, which also leads to a substantial simplification of the dynamics of the particle system. However, the magnitude of the dipole moment, and, hence, the strength of interaction of the components cannot be continuously modulated.

Analyzing the motion of a single particle in the dipole field of another one, we showed that the motion of particles is deterministic and can be described in terms of Stokes drag and the pointlike dipole approximation. Fluctuations such as surface waves of the carrier liquid generated by the moving particles do not affect the time evolution of the particle system due to the stabilizing effect of the relatively large swimmers (cork disks). Experiments were performed varying the total concentration of the particles and the relative concentration and relative dipole moment of the components. At low concentrations, cluster-cluster aggregation was observed with chainlike morphologies, while at higher concentrations, a multitude of planar crystal lattices was obtained: the particles formed triangular lattices of a single component, binary honeycomb and square lattices, and hexagonal closed packed structures The dynamics of the aggregation process, the structure of particle clusters and the parameter regimes of their occurrence are in a satisfactory agreement with recent theoretical predictions [27]. The comparison to the model calculations also revealed the fragility of structures which allows the compaction of particle clusters in subsequent rearrangements resulting in configurations of lower energy. The stabilizing effect of static friction arising at the contact surface of particles inside clusters proved to be an important element of the structure formation. Our study provided the first experimental realization of the binary honeycomb lattice where both types of particles have three neighbors of the other one. Based on our results, it is reasonable to assume that in former experiments on binary dipolar monolayers with ac electric fields, the complicated electrohydrodynamic flow of the embedding liquid could inhibit the formation of structures with long range order but low packing fraction like the honeycomb lattice.

Due to its simplicity, the experimental technique has a broad range of potential applications. For instance, changing also the shape of the container, the structure of clusters of dipolar particles in hard wall confining potentials could easily be studied [33].

\section{ACKNOWLEDGMENTS}

F.K. is grateful to the Japan Society for the Promotion of Science (JSPS) for the generous support during a research stay in Japan. F.K. was supported by Research Contracts No. OTKA T037212 and No. M041537, and by the György Békési Foundation of the Hungarian Academy of Sciences. This work was supported in the framework of a HungarianChinese Intergovernmental Project No. CHN-14/04. 
[1] A. T. Skjeltorp, Phys. Rev. Lett. 51, 2306 (1983).

[2] J. Cernák, G. Helgesen, and A. T. Skjeltorp, Phys. Rev. E 70, 031504 (2004).

[3] J. M. Tavares, J. J. Weis, and M. M. Teb da Gama, Phys. Rev. E 65, 061201 (2002).

[4] J. J. Weis, J. M. Tavares, and M. M. T. da Gama, J. Phys.: Condens. Matter 14, 9171 (2002).

[5] P. I. C. Teixeira, J. M. Tavares, and M. M. Telo da Gama, J. Phys.: Condens. Matter 12, R411 (2000).

[6] H. Morimoto, T. Maekawa, and Y. Matsumoto, Phys. Rev. E 68, 061505 (2003).

[7] A. Ghazali and J. C. Lévy, Phys. Rev. B 67, 064409 (2003).

[8] J. Y. Huang and P. Y. Lai, Physica A 281, 105 (2000).

[9] E. M. Furst and A. P. Gast, Phys. Rev. Lett. 82, 4130 (1999).

[10] E. M. Furst and A. P. Gast, Phys. Rev. E 61, 6732 (2000).

[11] W. Wen, F. Kun, K. F. Pál, D. W. Zheng, and K. N. Tu, Phys. Rev. E 59, R4758 (1999).

[12] F. Kun, K. F. Pál, Weijia Wen, and K. N. Tu, Phys. Lett. A 277, 287 (2000); F. Kun, Weijia Wen, K. F. Pál, and K. N. Tu, Phys. Rev. E 64, 061503 (2001).

[13] W. Wen, L. Zhang, and P. Sheng, Phys. Rev. Lett. 85, 5464 (2000).

[14] S. Yeh, M. Seul, and B. L. Shralman, Nature (London) 386, 57 (1997).

[15] Y. Choi, K. Kim, and H. K. Pak, Physica A 281, 99 (2000).

[16] C. Eisenmann, U. Gasser, P. Keim, and G. Maret, Phys. Rev. Lett. 93, 105702 (2004).

[17] Y. Terada and M. Tokuyama, Physica A 334, 327 (2004).
[18] L. E. Helseth and T. M. Fischer, Phys. Rev. E 68, 051403 (2003).

[19] W. Wen and P. Sheng, Physica B 338, 343 (2003).

[20] V. A. Froltsov, R. Blaak, C. N. Likos, and H. Löwen, Phys. Rev. E 68, 061406 (2003).

[21] K. Zahn, A. Wille, G. Maret, S. Sengupta, and P. Nielaba, Phys. Rev. Lett. 90, 155506 (2003).

[22] A. Chakrabarti, D. Fry, and C. M. Sorensen, Phys. Rev. E 69, 031408 (2004)

[23] B. A. Grzybowski, X. Jiang, H. A. Stone, and G. M. Whitesides, Phys. Rev. E 64, 011603 (2004).

[24] P. D. Duncan and P. J. Camp, J. Chem. Phys. 121, 11322 (2004).

[25] S. Fazekas, J. Kertesz, and D. E. Wolf, Phys. Rev. E 68, 041102 (2003)

[26] W. D. Ristenpart, I. A. Aksay, and D. A. Saville, Phys. Rev. Lett. 90, 128303 (2003).

[27] I. Varga, F. Kun, and K. F. Pál, Phys. Rev. E 69, 030501(R) (2004).

[28] D. L. Blair and A. Kudrolli, Phys. Rev. E 67, 021302 (2003).

[29] J. Stambaugh, D. P. Lathrop, E. Ott, and W. Losert, Phys. Rev. E 68, 026207 (2003).

[30] J. Stambaugh, Z. Smith, E. Ott, and W. Losert, Phys. Rev. E 70, 031304 (2004).

[31] T. Vicsek and F. Family, Phys. Rev. Lett. 52, 1669 (1984).

[32] N. Yoshioka et al. (unpublished).

[33] M. Kong, B. Partoens, A. Matulis, and F. M. Peeters, Phys. Rev. E 69, 036412 (2004). 